\title{
New bounds for Randic and GA indices
}

\author{
V Lokesha' ${ }^{1}$ B Shwetha Shetty ${ }^{2}$, PS Ranjini ${ }^{3}$, Ismail Naci Cangu ${ }^{4^{*}}$ and Ahmet Sinan Cevik ${ }^{5}$ \\ Dedicated to Professor Hari M Srivastava.
}

\section{"Correspondence:}

cangul@uludag.edu.tr

${ }^{4}$ Department of Mathematics,

Faculty of Arts and Science, Uludag

University, Gorukle Campus, Bursa,

16059, Turkey

Full list of author information is

available at the end of the article

\begin{abstract}
The main goal of this paper is to present some new lower and upper bounds for the Randic and GA indices in terms of Zagreb and modified Zagreb indices.

MSC: 05C05; 05C20; 05C90
\end{abstract}

Keywords: Randic index; GA index; Zagreb index; modified Zagreb index

\section{Introduction and preliminaries}

A systematic study of topological indices is one of the most striking aspects in many branches of mathematics with its applications and various other fields of science and technology. A topological index is a numeric quantity from the structural graph of a molecule. Usage of topological indices in chemistry began in 1947 when H. Wiener developed the most widely known topological descriptor, namely the Wiener index, and used it to determine physical properties of types of alkanes known as paraffin (see, for instance, [1-3]).

Let $G$ be a simple graph with the vertex-set $V(G)$ and the edge-set $E(G)$. As usual notion, the maximum vertex degree is denoted by $\Delta=\Delta(G)$, while the minimum vertex degree is denoted by $\delta=\delta(G)$. Moreover, $\delta_{1}=\delta_{1}(G)$ denotes the minimum nonpendant vertex degree in $G$. A vertex of the graph $G$ is said to be pendant if its neighborhood contains exactly one vertex. On the other hand, an edge of a graph is said to be pendant if one of its vertices is pendant.

In 1975, Randic [4] introduced the connectivity index, namely Randic index, to reflect molecular branching. In fact, the Randic index is defined as

$$
\chi(G)=\sum_{u v \in E(G)} \frac{1}{\sqrt{d_{u} d_{v}}}
$$

Furthermore, again by considering the degrees of vertices in G, Vukicević and Furtula [5] developed the Geometric-arithmetic index, shortly GA index, which is defined by

$$
\mathrm{GA}(G)=\sum_{u v \in E(G)} \frac{2 \sqrt{d_{u} d_{v}}}{d_{u}+d_{v}}
$$

In the following, we recall two fundamental indices that will be used to present some new bounds for Randic and GA indices.

(C) 2013 Lokesha et al.; licensee Springer. This is an Open Access article distributed under the terms of the Creative Commons Attribution License (http://creativecommons.org/licenses/by/2.0), which permits unrestricted use, distribution, and reproduction in any medium, provided the original work is properly cited. 
The (first and second) Zagreb indices have been introduced by Gutman and Trinajstić [6] as the form

$$
M_{1}(G)=\sum_{v \in V(G)}\left(d_{v}\right)^{2} \quad \text { and } \quad M_{2}(G)=\sum_{u v \in E(G)} d_{u} d_{v}
$$

where $d_{u}$ and $d_{v}$ are the degrees of $u$ and $v$, respectively. On the other hand, for a (molecular) graph $G$, the modified second Zagreb index $M_{2}^{*}(G)$ is defined as

$$
M_{2}^{*}(G)=\sum_{u v \in E(G)} \frac{1}{d_{u} d_{v}}
$$

(cf. [7-10]).

This paper is organized as follows. In the forthcoming section, we present lower and upper bounds on Randic index of connected graphs and trees in terms of modified Zagreb indices given in (4). The final section deals with lower and upper bounds on GA index of connected graphs and trees in terms of Zagreb indices given in (3). We note that this paper is motivated from [11].

\section{Lower and upper bounds on Randic index}

Throughout this paper, we refer the book [12] for a classical result, namely the PólyaSzegó inequality. From this result, we first establish the following theorem, which will be expressed the lower bound on the Randic index.

Theorem 1 Let $G$ be a simple connected graph of order $n$ with $m$ edges, and let $p, \Delta$ and $\delta_{1}$ denote the number of pendant vertices, maximum vertex degree and minimum nonpendant vertex degree of $G$, respectively. Then

$$
\chi(G) \geq \frac{p}{\sqrt{\Delta}}+\frac{2 \sqrt{\delta_{1} \Delta(m-p)}}{\delta_{1}+\Delta} \sqrt{M_{2}^{*}(G)-\frac{p}{\Delta}} .
$$

Proof For $2 \leq \delta_{1} \leq d_{i}, d_{j} \leq \Delta$, we clearly have

$$
\frac{1}{d_{i} d_{j}} \geq \frac{1}{d_{i} \Delta} \geq \frac{1}{\Delta^{2}}
$$

such that the equality holds if and only if $d_{i}=d_{j}=\Delta$. We also have

$$
\frac{1}{d_{i} d_{j}} \leq \frac{1}{d_{i} \delta_{1}} \leq \frac{1}{\delta_{1}^{2}}
$$

with equality holding if and only if $d_{i}=d_{j}=\delta_{1}$.

Since $p$ is the number of pendant vertices in $G$, we have total $m-p$ number of nonpendant edges in $G$. By the Pólya-Szegó inequality, we have

$$
\begin{aligned}
\left(\sum_{v_{i} v_{j} \in E(G): d_{j}, d_{j} \neq 1} \frac{1}{\sqrt{d_{i} d_{j}}}\right)^{2} & \geq \frac{4 \delta_{1} \Delta(m-p)}{\left(\delta_{1}+\Delta\right)^{2}}\left(\sum_{v_{i} v_{j} \in E(G): d_{i}, d_{j} \neq 1} \frac{1}{d_{i} d_{j}}\right) \\
& \geq \frac{4 \delta_{1} \Delta(m-p)}{\left(\delta_{1}+\Delta\right)^{2}}\left(M_{2}^{*}(G)-\sum_{v_{i} v_{j} \in E(G): d_{i}=1} \frac{1}{d_{j}}\right) .
\end{aligned}
$$


This inequality can be clearly written as

$$
\sum_{v_{i} \nu_{j} \in E(G): d_{j}, d_{j} \neq 1} \frac{1}{\sqrt{d_{i} d_{j}}} \geq \frac{\sqrt{4 \delta_{1} \Delta(m-p)}}{\left(\delta_{1}+\Delta\right)} \sqrt{M_{2}^{*}(G)-p \frac{1}{\Delta}} .
$$

From (1), we get

$$
\chi(G)=\sum_{v_{i} v_{j} \in E(G): d_{i}=1} \frac{1}{\sqrt{d_{j}}}+\sum_{v_{i} v_{j} \in E(G): d_{i}, d_{j} \neq 1} \frac{1}{\sqrt{d_{i} d_{j}}} .
$$

For $\Delta \geq d_{i}$, since $\frac{1}{d_{i}} \geq \frac{1}{\Delta}$, by (5) and (6), we obtain

$$
\chi(G) \geq \frac{p}{\sqrt{\Delta}}+\frac{2 \sqrt{\delta_{1} \Delta(m-p)}}{\delta_{1}+\Delta} \sqrt{M_{2}^{*}(G)-\frac{p}{\Delta}},
$$

as desired.

Corollary 1 Let $T$ be a tree of order $n$ with $p$ pendant vertices, and let $\Delta$ and $\delta_{1}$ be the maximum vertex and minimum nonpendent vertex degrees of $T$, respectively. Then

$$
\chi(T) \geq \frac{p}{\sqrt{\Delta}}+\frac{2 \sqrt{\delta_{1} \Delta(n-1-p)}}{\delta_{1}+\Delta} \sqrt{M_{2}^{*}(G)-\frac{p}{\Delta}} .
$$

Proof Since the number of edges in a tree having $n$ vertices is $m=n-1$, the proof can be done similarly as in the proof of Theorem 1.

Theorem 2 Let $G$ be a simple connected graph of order $n$ with $m$ edges, and let $p, \Delta$ and $\delta_{1}$ denote the number of pendant vertices, maximum vertex degree and minimum nonpendant vertex degree of $G$, respectively. Then

$$
\chi(G) \leq \frac{p}{\sqrt{\delta_{1}}}+\sqrt{(m-p)\left(M_{2}^{*}(G)-\frac{p}{\delta_{1}}\right)} .
$$

Proof By the Cauchy-Schwarz inequality, it is clear that

$$
\begin{aligned}
\left(\sum_{v_{i} \nu_{j} \in E(G): d_{j}, d_{j} \neq 1} \frac{1}{\sqrt{d_{i} d_{j}}}\right)^{2} & \leq(m-p)\left(\sum_{v_{i} v_{j} \in E(G): d_{j}, d_{j} \neq 1} \frac{1}{d_{i} d_{j}}\right) \\
& \leq(m-p)\left(M_{2}^{*}(G)-\sum_{v_{i} v_{j} \in E(G): d_{i}=1} \frac{1}{d_{j}}\right) \\
& \leq(m-p)\left(M_{2}^{*}(G)-\frac{p}{\delta_{1}}\right)
\end{aligned}
$$

which can be rewritten as

$$
\sum_{v_{i} \nu_{j} \in E(G): d_{j}, d_{j} \neq 1} \frac{1}{\sqrt{d_{i} d_{j}}} \leq \sqrt{(m-p)\left(M_{2}^{*}(G)-\frac{p}{\delta_{1}}\right)} .
$$


Since $\frac{1}{d_{j}} \leq \frac{1}{\delta_{1}}$ for $\delta_{1} \leq d_{j}$, by (5) and (7), we obtain

$$
\chi(G) \leq \frac{p}{\sqrt{\delta_{1}}}+\sqrt{(m-p)\left(M_{2}^{*}(G)-\frac{p}{\delta_{1}}\right)}
$$

as required.

Now we prove another form of the upper bound for the Randic index as in the following.

Theorem 3 Let $G$ be a simple connected graph of order $n$ with $m$ edges, and let $p, \Delta$ and $\delta_{1}$ denote the number of pendant vertices, maximum vertex degree and minimum nonpendant vertex degree of $G$, respectively. Then

$$
\chi(G) \leq \frac{p}{\sqrt{\delta_{1}}}+\frac{(m-p)}{\delta_{1}}
$$

Proof Since $\frac{1}{\delta_{1}^{2}}$ is the maximum value of $\frac{1}{d_{i} d_{j}}$ for all edges $v_{i} v_{j} \in E(G)$, we have

$$
\begin{aligned}
M_{2}^{*}(G)-\sum_{v_{i} v_{j} \in E(G): d_{i}=1} \frac{1}{d_{j}} & =\sum_{v_{i} v_{j} \in E(G): d_{j}, d_{j} \neq 1} \frac{1}{\sqrt{d_{i} d_{j}}} \\
& \leq \frac{m-p}{\delta_{1}^{2}} .
\end{aligned}
$$

After that, by using (9) in (5), we get the bound in (8), as required.

\section{Lower and upper bounds on GA index}

By taking Pólya-Szegó inequality into account, the next result deals with a new lower bound on GA index in terms of Zagreb index as given in (3).

Theorem 4 Let $G$ be a simple connected graph of order $n$ with $m$ edges, and let $p, \Delta$ and $\delta_{1}$ denote the number of pendant vertices, maximum vertex degree and minimum nonpendant vertex degree of $G$, respectively. Then

$$
\mathrm{GA}(G) \geq \frac{2 p \sqrt{\delta_{1}}}{1+\Delta}+2 \sqrt{2} \frac{\delta_{1} \Delta}{\left(\delta_{1}^{2}+\Delta^{2}\right)} \sqrt{\frac{(m-p)}{\Delta}\left(M_{2}(G)-p \delta_{1}\right)}
$$

Proof For $2 \leq \delta_{1} \leq d_{i}, d_{j} \leq \Delta$, we have

$$
\frac{1}{2 \Delta} \leq \frac{1}{\left(d_{i}+d_{j}\right)} \leq \frac{1}{2 \delta_{1}}
$$

which implies

$$
\frac{d_{i} d_{j}}{\left(d_{i}+d_{j}\right)^{2}} \leq \frac{\Delta^{2}}{4 \delta_{1}^{2}}
$$

On the other hand, since we also have

$$
\frac{d_{i} d_{j}}{\left(d_{i}+d_{j}\right)^{2}} \geq \frac{\delta_{1}^{2}}{4 \Delta^{2}}
$$


the combination of these above equalities implies that

$$
\frac{\delta_{1}}{\Delta} \leq \frac{2 \sqrt{d_{i} d_{j}}}{\left(d_{i}+d_{j}\right)} \leq \frac{\Delta}{\delta_{1}}
$$

Since $p$ is the number of pendant vertices in $G$, we have total $m-p$ number of nonpendant edges in G. By the Pólya-Szegó inequality, we get

$$
\begin{aligned}
\left(\sum_{v_{i} v_{j} \in E(G): d_{j}, d_{j} \neq 1} \frac{2 \sqrt{d_{i} d_{j}}}{\left(d_{i}+d_{j}\right)}\right)^{2} & \geq \frac{4 \delta_{1}^{2} \Delta^{2}(m-p)}{\left(\delta_{1}^{2}+\Delta^{2}\right)^{2}}\left(\sum_{v_{i} v_{j} \in E(G): d_{i}, d_{j} \neq 1} \frac{4 d_{i} d_{j}}{\left(d_{i}+d_{j}\right)}\right) \\
& \geq \frac{4 \delta_{1}^{2} \Delta^{2}(m-p)}{\left(\delta_{1}^{2}+\Delta^{2}\right)^{2}}\left(\sum_{v_{i} v_{j} \in E(G): d_{i}, d_{j} \neq 1} \frac{4 d_{i} d_{j}}{2 \Delta}\right) \\
& \geq \frac{8 \delta_{1}^{2} \Delta^{2}(m-p)}{\Delta\left(\delta_{1}^{2}+\Delta^{2}\right)^{2}}\left(M_{2}(G)-\sum_{v_{i} v_{j} \in E(G): d_{i}=1} d_{j}\right) \\
& \geq \frac{8 \delta_{1}^{2} \Delta^{2}(m-p)}{\Delta\left(\delta_{1}^{2}+\Delta^{2}\right)^{2}}\left(M_{2}(G)-p \delta_{1}\right) .
\end{aligned}
$$

This calculation can be rewritten basically as follows:

$$
\sum_{v_{i} v_{j} \in E(G): d_{j}, d_{j} \neq 1} \frac{2 \sqrt{d_{i} d_{j}}}{\left(d_{i}+d_{j}\right)} \geq 2 \sqrt{2} \frac{\delta_{1} \Delta}{\left(\delta_{1}^{2}+\Delta^{2}\right)} \sqrt{\frac{(m-p)}{\Delta}\left(M_{2}(G)-p \delta_{1}\right)}
$$

From (2), we obtain

$$
\mathrm{GA}(G)=\sum_{v_{i} v_{j} \in E(G): d_{i}=1} \frac{2 \sqrt{d_{j}}}{\left(1+d_{j}\right)}+\sum_{v_{i} v_{j} \in E(G): d_{i}, d_{j} \neq 1} \frac{2 \sqrt{d_{i} d_{j}}}{\left(d_{i}+d_{j}\right)}
$$

Now, for $\delta_{1} \leq d_{j} \leq \Delta$, since $\sqrt{d_{j}} \geq \sqrt{\delta_{1}}$ and $\frac{1}{1+d_{j}} \geq \frac{1}{1+\Delta}$, by (10) and (11), we arrive at

$$
\mathrm{GA}(G) \geq \frac{2 p \sqrt{\delta_{1}}}{1+\Delta}+2 \sqrt{2} \frac{\delta_{1} \Delta}{\left(\delta_{1}^{2}+\Delta^{2}\right)} \sqrt{\frac{(m-p)}{\Delta}\left(M_{2}(G)-p \delta_{1}\right)} .
$$

Hence the result.

Corollary 2 Let $T$ be a tree of order $n$ with $p$ pendant vertices, and let $\Delta$ and $\delta_{1}$ denote the maximum vertex degree and minimum non-pendent vertex degree of $T$, respectively. Then

$$
\mathrm{GA}(G) \geq \frac{2 p \sqrt{\delta_{1}}}{1+\Delta}+2 \sqrt{2} \frac{\delta_{1} \Delta}{\left(\delta_{1}^{2}+\Delta^{2}\right)} \sqrt{\frac{(n-1-p)}{\Delta}\left(M_{2}(G)-p \delta_{1}\right)}
$$

Proof For an order $n$, since the number of edges in a tree $T$ is $m=n-1$, the proof can be done quite similar as the proof of Theorem 4.

Theorem 5 Let $G$ be a simple connected graph of order $n$ with $m$ edges, and let $p, \Delta$ and $\delta_{1}$ denote the number of pendant vertices, maximum vertex degree and minimum non- 
pendant vertex degree of $G$, respectively. Then

$$
\mathrm{GA}(G) \leq \frac{2 p \sqrt{\Delta}}{1+\delta_{1}}+\frac{1}{\delta_{1}} \sqrt{(m-p)\left(M_{2}(G)-p \Delta\right)}
$$

Proof By the Cauchy-Schwarz inequality,

$$
\begin{aligned}
\left(\sum_{v_{i} v_{j} \in E(G): d_{j}, d_{j} \neq 1} \frac{2 \sqrt{d_{i} d_{j}}}{\left(d_{i}+d_{j}\right)}\right)^{2} & \leq(m-p)\left(\sum_{v_{i} v_{j} \in E(G): d_{j}, d_{j} \neq 1} \frac{4 d_{i} d_{j}}{\left(d_{i}+d_{j}\right)^{2}}\right) \\
& \leq(m-p)\left(\sum_{v_{i} v_{j} \in E(G): d_{j}, d_{j} \neq 1} \frac{d_{i} d_{j}}{\delta_{1}^{2}}\right) \\
& \leq \frac{(m-p)}{\delta_{1}^{2}}\left(M_{2}(G)-\sum_{v_{i} v_{j} \in E(G): d_{i}=1} d_{j}\right) \\
& \leq \frac{(m-p)}{\delta_{1}^{2}}\left(M_{2}(G)-p \Delta\right)
\end{aligned}
$$

which can be simply indicate as

$$
\sum_{v_{i} v_{j} \in E(G): d_{j}, d_{j} \neq 1} \frac{2 \sqrt{d_{i} d_{j}}}{\left(d_{i}+d_{j}\right)} \leq \frac{1}{\delta_{1}} \sqrt{(m-p)\left(M_{2}(G)-p \Delta\right)} .
$$

Now, for $\delta_{1} \leq d_{j} \leq \Delta$, since $\sqrt{d_{j}} \leq \sqrt{\Delta}$ and $\frac{1}{1+d_{j}} \leq \frac{1}{1+\delta_{1}}$, by (11) and (12) we get the result, as required.

The following theorem presents another upper bound for GA index.

Theorem 6 Let $G$ be a simple connected graph of order $n$ with $m$ edges, and let $p, \Delta$ and $\delta_{1}$ denote the number of pendant vertices, maximum vertex degree and minimum nonpendant vertex degree of $G$, respectively. Then

$$
G(A) \leq \frac{2 p \sqrt{\Delta}}{1+\delta_{1}}+\frac{(m-p) \Delta}{\delta_{1}}
$$

Proof Since $\Delta^{2}$ is the maximum value of $d_{i} d_{j}$ for all edges $v_{i} v_{j} \in E(G)$, we have

$$
\begin{aligned}
M_{2}(G)-\sum_{v_{i} v_{j} \in E(G): d_{i}=1} d_{j} & =\sum_{v_{i} v_{j} \in E(G): d_{j}, d_{j} \neq 1} d_{i} d_{j} \\
& \leq(m-p) \Delta^{2} .
\end{aligned}
$$

Now, by using (13) in (11), we get

$$
G(A) \leq \frac{2 p \sqrt{\Delta}}{1+\delta_{1}}+\frac{(m-p) \Delta}{\delta_{1}} .
$$

Hence, the result. 


\section{Competing interests}

The authors declare that they have no competing interests.

\section{Authors' contributions}

All authors completed the paper together. All authors read and approved the final manuscript.

\section{Author details}

${ }^{1}$ Department of Mathematics, V S K University, Bellary, 583 104, India. ²Department of Mathematics, Sri Krishna School of Engineering and Management, Bangalore, 35, India. ${ }^{3}$ Department of Mathematics, Don Bosco Institute of Technology, Bangalore, 78, India. ${ }^{4}$ Department of Mathematics, Faculty of Arts and Science, Uludag University, Gorukle Campus, Bursa, 16059, Turkey. ${ }^{5}$ Department of Mathematics, Faculty of Science, Selcuk University, Campus, Konya, 42075, Turkey.

\section{Acknowledgements}

I.N. Cangul and A.S. Cevik are partially supported by Research Project Offices of Uludag (2012-15, 2012-19 and 2012-20) and Selcuk Universities, respectively.

Received: 9 February 2013 Accepted: 26 March 2013 Published: 16 April 2013

\section{References}

1. Estrada, E, Torres, L, Rodriguez, L, Gutman, I: An atom-bond connectivity index. Modelling the enthalpy of formation of alkanes. Indian J. Chem. 37A, 849-855 (1998)

2. Ranjini, PS, Lokesha, V, Bindusree, M, Phani Raju, M: New bounds on Zagreb indices and the Zagreb co-indices. Bol. Soc. Parana. Mat. SPM. ISSN-2175-1188 online, SPM: www.spm.uem.br/bspm

3. Trinajstić, N: Chemical Graph Theory. CRC Press, Boca Raton (1992)

4. Randic, M: Characterization of molecular branching. J. Am. Chem. Soc. 97, 6609-6615 (1975)

5. Vukicević, D, Furtula, B: Topological index based on the ratios of geometrical and arithmetical means of end-vertex degrees of edges. J. Math. Chem. 4, 1369-1376 (2009)

6. Gutman, I, Trinajstić, N: Graph theory and molecular orbitals. Total $\frac{1}{4}$-electron energy of alternate hydrocarbons. Chem. Phys. Lett. 17, 535-538 (1972)

7. Das, KC, Gutman, I, Zhou, B: New upper bounds on Zagreb indices. J. Math. Chem. 46, 514-521 (2009)

8. Ranjini, PS, Lokesha, V: The Smarandache-Zagreb indices on the three graph operators. Int. J. Math. Combin. China 3, 1-10 (2010)

9. Ranjini, PS, Lokesha, V, Cangul, IN: On the Zagreb indices of the line graphs of the subdivision graphs. Appl. Math. Comput. 218(3), 699-702 (2011). doi:10.1016/j.amc.2011.03.125

10. Ranjini, PS, Lokesha, V, Rajan, MA: On Zagreb indices of the subdivision graphs. Int. J. Math. Sci. Eng. Appl 4, 221-228 (2010)

11. Das, KC: Atom-bond connectivity index of graphs. Discrete Appl. Math. 158, 1181-1188 (2010)

12. Pólya, G, Szegó, G: Problems and Theorems in Analysis I: Series, Integral Calculus, Theory of Functions. Springer, New York (1972)

doi:10.1186/1029-242X-2013-180

Cite this article as: Lokesha et al.: New bounds for Randic and GA indices. Journal of Inequalities and Applications 2013 2013:180.

\section{Submit your manuscript to a SpringerOpen ${ }^{\circ}$ journal and benefit from:}

- Convenient online submission

Rigorous peer review

- Immediate publication on acceptance

- Open access: articles freely available online

- High visibility within the field

- Retaining the copyright to your article 